\title{
The European Spallation Source
}

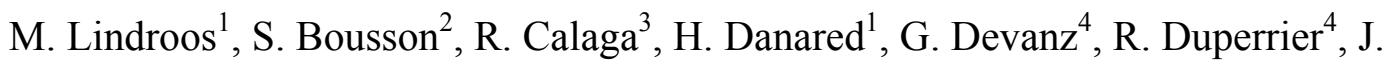
Eguia $^{5}$, M. Eshraqi ${ }^{1}$, S. Gammino ${ }^{6}$, H. Hahn ${ }^{1}$, A. Jansson ${ }^{1}$, C. Oyon ${ }^{7}$,

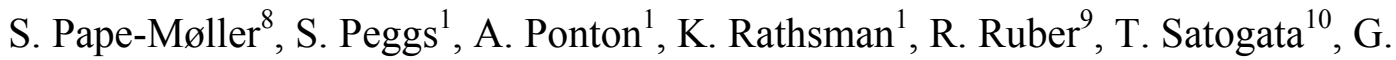
Trahern $^{1}$

${ }^{1}$ ESS AB, Lund, Sweden

${ }^{2} I P N O$, CNRS/IN2P3, Orsay, France

${ }^{3}$ BNL, Brookhaven National Laboratory, Upton, NY, USA

${ }^{4}$ CEA-Saclay, Gif sur Yvette, France

${ }^{5}$ Tekniker, Eibar-Guipuzcoa, Spain

${ }^{6} I N F N-L N S$, Catania, Italy,

${ }^{7}$ SPRI, Bilbao, Spain

${ }^{8}$ Aarhus University, Ny Munkegade, Aarhus C, Denmark

${ }^{9}$ Uppsala University, Uppsala, Sweden

${ }^{10}$ Jefferson Lab, Newport News, VA, USA

\section{ABSTRACT}

In 2003 the joint European effort to design a European Spallation Source (ESS)

resulted in a set of reports, and in May 2009 Lund was agreed to be the ESS site. The ESS Scandinavia office has since then worked on setting all the necessary legal and organizational matters in place so that the Design Update and construction can be started in January 2011, in collaboration with European partners. The Design Update phase is expected to end in 2012 , to be followed by a construction phase, with first neutrons expected in 2018-2019. 


\section{INTRODUCTION}

Table 1 shows the two tentative sets of primary ESS parameters that were presented at the ESS-Bilbao Initiative Workshop, in March 2009 [1]. Columns B and S show theclose similarity between the parameters of the Bilbao and Scandinavia designs. In many cases the values are identical. Where they do deviate, the differences are relatively minor. In contrast, the average beam current and the final beam energy differ by at least a factor of 2 from the 2003 ESS design values ( $5 \mathrm{MW}, 1 \mathrm{GeV}, 150$ $\mathrm{mA}, 16.7 \mathrm{~Hz}$ ) [2]. Decreasing the beam current and increasing the beam energy simplifies the linac design and increases the reliability. Decreasing the beam current allows the cavity gradient to increase (at fixed power coupler strength), but keeps the linac length approximately unchanged from the 2003 values, despite the increase in beam energy.

The Design Update phase that begins in January 2011 will resolve most of the design issues and many of the lower level design parameters, for reporting in the Conceptual Design Report that will be delivered in December 2012. However, high level parameters and decisions that will hold their validity for at least 2 years need to be established even before that design effort can proceed. We have therefore committed to defining a DU Baseline by the end of December 2010. The issues affecting the evolution of this baseline from the current "provisional baseline" are presented below, together with a description of some work already performed, tentative conclusions already drawn for the control system, and an outline of future work. (For simplicity, column $\mathrm{S}$ values in Table 1 are taken to be the provisional baseline values, if B and $\mathrm{S}$ values differ.) 
Table 1: Primary ESS performance parameters in the long pulse conceptual design.

Columns B and S show the minor differences between the ESS-Bilbao and ESS-

Scandinavia nominal parameters (2009).

\begin{tabular}{|lcccc|}
\hline & & & & S \\
INPUT & & & & \\
Average beam power & {$[\mathrm{MW}]$} & & 5.0 & \\
No. of instruments & & & 22 & \\
Macro-pulse length & {$[\mathrm{ms}]$} & 1.5 & & 2.0 \\
Pulse repetition rate & {$[\mathrm{Hz}]$} & & 20 & \\
Proton kinetic energy & {$[\mathrm{GeV}]$} & 2.2 & & 2.5 \\
Peak coupler power & {$[\mathrm{MW}]$} & 1.2 & & 1.0 \\
Beam loss rate & {$[\mathrm{W} / \mathrm{m}]$} & & $<1.0$ & \\
& & & & \\
OUTPUT & & & & \\
Duty factor & & 0.03 & & 0.04 \\
Ave. current on target & {$[\mathrm{mA}]$} & 2.3 & & 2.0 \\
Ave. pulse current & {$[\mathrm{mA}]$} & 75 & & 50 \\
Ion source current & {$[\mathrm{mA}]$} & $\sim 90$ & & 60 \\
Total linac length & {$[\mathrm{m}]$} & & $\sim 420$ & \\
& & & & \\
\hline
\end{tabular}

\section{ESS ACCELERATOR DESIGN UPDATE PROJECT}

The ESS Accelerator Design Update (ADU) will be performed within a European collaboration structure with eight work packages: 1. Management, 2. Accelerator Science, 3. Infrastructure and services, 4. Spoke SCRF, 5. Elliptical SCRF, 6. Normal conducting front-end, 7. High Energy Beam Transport, magnets and power supplies and 8. RF systems. Each work package has been planned by one major European Institute/University, with the first and second being lead by ESS. Contributions from multiple institutes and Universities are expected in all work packages. The ADU project is planned to start 1 January 2011, with in-kind contributions from participating countries for staff and prototypes. 


\section{DESIGN UPDATE BASELINE}

The evolution to the DU baseline requires fixing parameters at reasonable values, and requires the statement of design decisions and philosophies.

\subsection{User parameters and potential upgrades.}

The provisional repetition rate is $20 \mathrm{~Hz}$, with a macropulse length of $2.0 \mathrm{~ms}$ that is acceptable to most of the neutron user community. All users want high availability few beam trips - in dynamic tension (for example) with an increase in beam current that could be necessary with shorter pulses. It is impossible to derive the availability of an ESS design from first principles, although there is empirical evidence from ISIS, LANSCE, PSI and SNS that the cumulative probability distribution of trip rate versus trip length follows a universal power law for trips of less than one day in duration [3, 4].

The DU baseline will be optimised for a nominal beam power of $5 \mathrm{MW}$, with a provisional current of $50 \mathrm{~mA}$ and a provisional peak power of $0.9 \mathrm{MW}$ in the elliptical cavity power coupler. This is consistent with the strategic philosophy

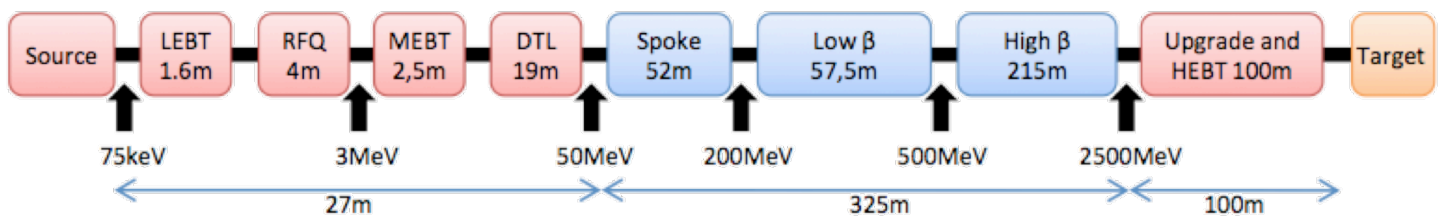

Figure 1: Provisional block layout of the ESS LINAC, not scaled.

that upgrade options will be preserved where reasonably possible. For example, the beam power may later be upgraded to $7.5 \mathrm{MW}$ by increasing the average current to 
$75 \mathrm{~mA}$ and adding extra cryomodules in the "Upgrade and HEBT" section shown schematically in Figure 1. Other potential upgrade options will also be studied.

2.2 Transition energies and beamline components.

Figure 1 and Table 2 show the provisional block layout of the linac, its transition energies between RF structure technologies, and the count of major components such as RF tanks and cryomodules. The transition energies may be further optimised for the DU baseline lattice, including the energy of the frequency jump between spoke resonators and elliptical cavities [5]. A more accurate representation of the ESS layout - for example, its length and its component counts - requires the inclusion of a full complement of beam instrumentation, collimation, magnets, correction systems, et cetera.

2.3 RF frequencies.

Two frequencies will be used in the normal and superconducting RF structures, 352.21 MHz and 704.42 MHz, the same frequencies that were selected for the CERN Linac4 and SPL [6]. The ESS Frequency Advisory Board report (2010) [7] endorses this choice.

Table 2: Provisional block layout of RF structures. 


\begin{tabular}{|lrcrr|}
\hline Structure & $\begin{array}{r}\text { Energy } \\
{[\mathrm{MeV}]}\end{array}$ & $\begin{array}{c}\text { Freq. } \\
{[\mathrm{MHz}]}\end{array}$ & Count & $\begin{array}{r}\text { Length } \\
{[\mathrm{m}]}\end{array}$ \\
& & & & \\
Source & 0.075 & - & - & 2.5 \\
LEBT & 0.075 & - & - & 1.6 \\
RFQ & 3 & 352.21 & 1 & 4.0 \\
MEBT & 3 & - & - & 2.5 \\
DTL & 50 & 352.21 & 3 & 19.0 \\
Spokes & 200 & 352.21 & 16 & 52.0 \\
Elliptical 1 & 500 & 704.42 & 9 & 57.0 \\
Elliptical 2 & 2500 & 704.42 & 14 & 215.0 \\
& & & & \\
\hline
\end{tabular}

\subsection{Prototype cryomodules.}

Circular superconducting accelerators have very few warm-to-cold transitions, usually connecting one magnet to the next with cold "spool pieces". In contrast, every cryomodule in the Spallation Neutron Source (SNS) linac is completely cryogenically "segmented" from its neighbor. Some superconducting linacs - those that are constrained in real estate length, such as the XFEL and the ILC - are designed with very little segmentation.

At ESS the two major technical drivers that will influence the level of cryogenic segmentation are the need to minimise the total site power through efficient energy engineering [8] and the need for high reliability (minimizing the down time due to failed cavities). A preliminary study suggests that a fully segmented linac would have 1.6 to 1.7 times the cryogenic power load of a fully non-segmented linac [9]. There are other more minor technical issues for the ESS, such as minimising the linac 
length, the risk of accidental contamination, and the desirability of de-coupling the development and integration of magnets and beam instrumentation from SRF development.

The construction and testing of prototype elliptical cavity cryomodules is a crucial part of the Design Update phase, in an effort that will extend beyond the end of 2012. In all scenarios these prototypes will be designed to have static and dynamic heat loads that are as low as reasonably achievable. In some scenarios the prototype cryomodules could differ significantly from the production cryomodules, leaving open until later the decision on the level of cryogenic segmentation.

Also under consideration is the desirability of making ESS cryomodules "plugcompatible", consistent with the ILC philosophy, in order to make design integration easier across the collaboration. For example, this would make it easier to include cavities from different sources in the prototype cryomodules, and it would reduce the set of standard beam (and other) instrumentation, and magnets, that need to be developed [10]. It would also simplify the incorporation of cavities from multiple vendors in the production line cryomodules. Standard shipping containers have an inside length of approximately $12.03 \mathrm{~m}$ [11]. Insisting that elliptical cryomodules are shorter than this could limit them to 6 cavities, although 8 may still be possible. This, too, is a consideration in arriving at a DU baseline configuration.

2.5 RF system parameters.

Table 3 shows the provisional parameters for the RF structures. The 3 geometric betas (for the spoke resonators, low-energy, and high-energy elliptical cavities) depend 
strongly on the baseline macropulse current, but depend only weakly on subscenarios that leave module transition energies unchanged.

The values shown correspond to operation with a $50 \mathrm{~mA}$ beam, consistent with the philosophy of optimising for the nominal beam power of 5.0 MW. Error bars of approximately 0.01 indicates the small size of changes that may occur in the move to the DU baseline.

Spoke resonator and elliptical cavity designs will be optimized within the design update collaboration, after the determination of the geometric betas has been finalised, taking into account issues like Higher Order Mode suppression.

The provisional maximum operating voltages and gradients shown in Table 3 are somewhat relaxed, since linac performance is mainly constrained by power coupler throughput, rather than by voltage or gradient. However, these values do not include any headroom, which must be included not only to ensure robust routine operations, but also to ensure that the cavity-to-cavity fluctuations are minimised, maximising the longitudinal acceptance and decreasing transverse beam losses. Detailed modeling and simulation studies are required before headroom specifications will be possible for spoke and elliptical cavity production lines, and for operations.

Table 3: Provisional RF system parameters, optimised for the $50 \mathrm{~mA}$ nominal macropulse current. Voltages and gradients are the maximum operational values per cavity, with little or no headroom. 


\section{Structure Geometric Maximum Maximum beta voltage gradient $\beta_{G} \quad[\mathrm{MV}] \quad[\mathrm{MV} / \mathrm{m}]$}

$\begin{array}{lrrr}\text { Spokes } & 0.54 \pm 0.01 & 5.6 & 8.0 \\ \text { Elliptical 1 } & 0.67 \pm 0.01 & 10.1 & 14.1 \\ \text { Elliptical 2 } & 0.83 \pm 0.01 & 18.5 & 21.1\end{array}$

\section{WORK IN PROGRESS}

The ESS linac will as far as possible be based on components that have been developed and used elsewhere for high intensity proton sources. The only exception is the proposed use of spoke cavities for intermediate energies. The high intensity proton ion source at INFN in Catania, VIS [12], and at CEA in Saclay, SILHI [13], serves as early prototypes for the ESS ion source. Long term stability tests and reliability tests are presently being performed at both locations and a collaboration within the ESS DU project is being set-up to design and build the ESS source. A reliable RFQ for high currents is of great importance for the project and a four vane structure such as built within the IPHI [14] project at CEA-Saclay. The four vane structure has already demonstrated reliable operation with low losses at the LEDA [15] facility at Los Alamos national laboratories, USA. The new H- injector at CERN, LINAC 4 [16], is making use of a DRL which also could be used for ESS. Furthermore, the higher energy part could serve as a back-up for ESS if the spoke cavity technology proves difficult. A first spoke cavity with fast tuners in an accelerator like cryostat was built and operated without beam for the EURISOL 
Design Study at IPNO in Paris [17]. Several five cell elliptical cavity structure have been tested worldwide e.g. at CEA in Saclay [18].

\section{DISCUSSION}

The ESS ADU is making good progress. The plan is to complete the ADU for the end of 2012 so that construction can start and first protons can be delivered 2018-2019. This will require a phased approach to the complete engineering Design Report as tendering and construction of time critical components such as Klystrons and SC cavities will have to start as early as 2013-2014. The plan is to make maximum use of existing European infrastructure for both testing and construction - for example, the XFEL construction infrastructure.

\section{REFERENCES}

[1] "Conclusions Report of the ESS-Bilbao Initiative Workshop", 2009. http://www.workshop2009.essbilbao.com/cas/conclusions.aspx [2] "ESS Volume III Update: Technical report status", 2003.

[3] D. Findlay \& C. Plostinar, private communications, 2010.

[4] J. Galambos et al, CPL04, Proc. of Hadron Beams, Nashville, 2008. http://accelconf.web.cern.ch/AccelConf/HB2008/papers/cp104.pdf [5] R. Duperrier et al, PRST AB, 10, 084201, 2007. http://prstab.aps.org/abstract/PRSTAB/v10/ $\mathrm{i} 8 / \mathrm{e} 084201$

[6] F. Gerigk et al, CERN-AB-2008-064, 2008. http://cdsweb.cern.ch/record/1132793/files/CERN-AB-2008-064.pdf [7] M. Harrison et al, “ESS Frequency Advisory Board Report”, July 2010. 
[8] "New neutron source aims to be top in energy and environmental stewardship", Physics Today, p24, March 2010.

[9] A. Ponton, ESS-LD-TECHNOTE-0310AP, March 2010.

http://esss.se/linac/Tech_Notes/ESS-LD-TECHNOTE-0310AP.pdf

[10] R. Rimmer, private communications, 2010.

[11] See, for example, http://www.srinternational.com/standard_containers.htm

[12] S. Gammino, L. Celona, R. Miracoli, D. Mascali, G. Castro, G. Ciavola, F. Maimone, R. Gobin, O. Delferrière, G. Adroit, F. Senèe, Proc. 19th Workshop on ECR Ion Sources (MOPOT012), Grenoble, August 2010, to be published on Jacow [13] High intensity ECR ion source $\mathrm{H}+, \mathrm{D}+, \mathrm{H}-\ldots$ developments at CEA/Saclay R. Gobin et al, Rev.Sci.Instr 73 (2002)

[14] P-Y. Beauvais, "Recent evolutions in the design of the French high intensity proton injector (IPHI)", Proceedings of EPAC 2004, Lucerne, Switzerland [15] H. Vernon Smith et al, "Commissioning results from the low-energy demonstration accelerator (LEDA) Radio-Frequency Quadrupole (RFQ)”, EPAC 2000

[16] M. Vretenar, "Status of Linac4 construction at CERN", Linac conference 2010, Tsukuba, Japan

[17] S. Bousson\#, J.L. Biarrotte, J.M. Dufour, N. Gandolfo, T. Junquera, J.

Lesrel, L. Lukovac, F. Lutton, G. Martinet, G. Olry, A. Ponton, E. Rampnoux, H. Saugnac, P. Szott, "Spoke cavity developments for the EURISOL driver", Proceedings of the LINAC 2006 conference, Knoxville, USA and The EURISOL-DS Final Report, Edited by J. Cornell, 2009, www.eurisol.org 
[18] G. Devanz et al., "Stiffened Medium Beta 704 MHZ Elliptical Cavity for a Pulsed Proton Linac", Proc. of SRF07, Beijing, 2007 http://cern.ch/AccelConf/srf2007/PAPERS/TUP81.pdf and G. Devanz et al. "704 MHz High Power Coupler and Cavity Development for High Power Pulsed Proton Linacs", Proc of EPAC08, Genoa, 2008 